\section{The experience of challenging behaviours following severe TBI: A family perspective}

Adam McKay ${ }^{123}$, Sammi Tam³, Sue Sloan4, \& Jennie L. Ponsford ${ }^{23}$

'Department of Psychology, Epworth Healthcare, Melbourne, Australia

${ }_{3}^{3}$ School of Psychological Sciences Monash University Melbeuraren Anstitute, Melbourne, Australl

sSchool of Psychological Sciences, Monash Universiy
4 Osborn Sloan and Associates, Melbourne, Australia

\section{Introduction}

Challenging behaviours such as aggression, reduced initiation, and socia

inappropriateness are common after severe traumatic brain injuries (TBI). These behaviours are often the most significant impediment to activity participation after TBI.

Professionals play an important role in addressing behavioural issues in the early stages after TBI, but families often become the primary support for managing challenging behaviours once the person returns to community living.

Existing research into the family experience of challenging behaviour after TBI has been focussed on quantitative studies using measures developed from the perspective of professionals. This may not fully capture the family experience.

\section{Aim}

The aim of this study was to understand the family experience of supporting a person with challenging behaviours after severe TBI. Qualitative in depth interviews focussed on understanding:

- Behaviours families consider to be challenging

- The impact of challenging behaviours on participation as viewed by families

- Strategies used by families to support and cope with challenging behaviours

\section{Participants}

- Six female relatives of individuals with very severe TBI (9-23 years post injury) were recruited through professionals working with clients with challenging behaviour

- Relatives included four mothers, one sister, and one spouse. Relatives either lived with or regularly spent time in the family home of the person with TBI.

\section{Design, Procedure and Analysis}

- Family members participated in a face-to-face semi-structured interviews with a researcher and ranged in duration from $28-77$ minutes $(M=53.7)$. Interviews were recorded and transcribed.

- Family members were asked a series open ended questions relevant to each of the three study aims with prompts to encourage elaboration.

- Interview transcripts were analysed into themes using a six-phase iterative thematic analysis approach outlined by Braun and Clarke (2006) and with all authors cross checking codes and themes.
Results

1) What behaviours do families find challenging after TBI?

- Families reported a range of behaviours to be challenging after TBI:

$$
\begin{array}{ll}
\text { Verbal and physical aggression } & - \text { Repetitive behaviours } \\
\text { Socially inappropriate } & -
\end{array}
$$$$
\text { behavior }
$$

Families considered cognitive problems to be part of 'challenging behaviours'. Problems with memory, speed of thinking, and impulsivity were seen as underpinning issues such as aggression, social inappropriateness, and poor initiation.

- Families had a good understanding of factors that modified the presentation of behaviours including mood, fatigue, confidence, and the environment.
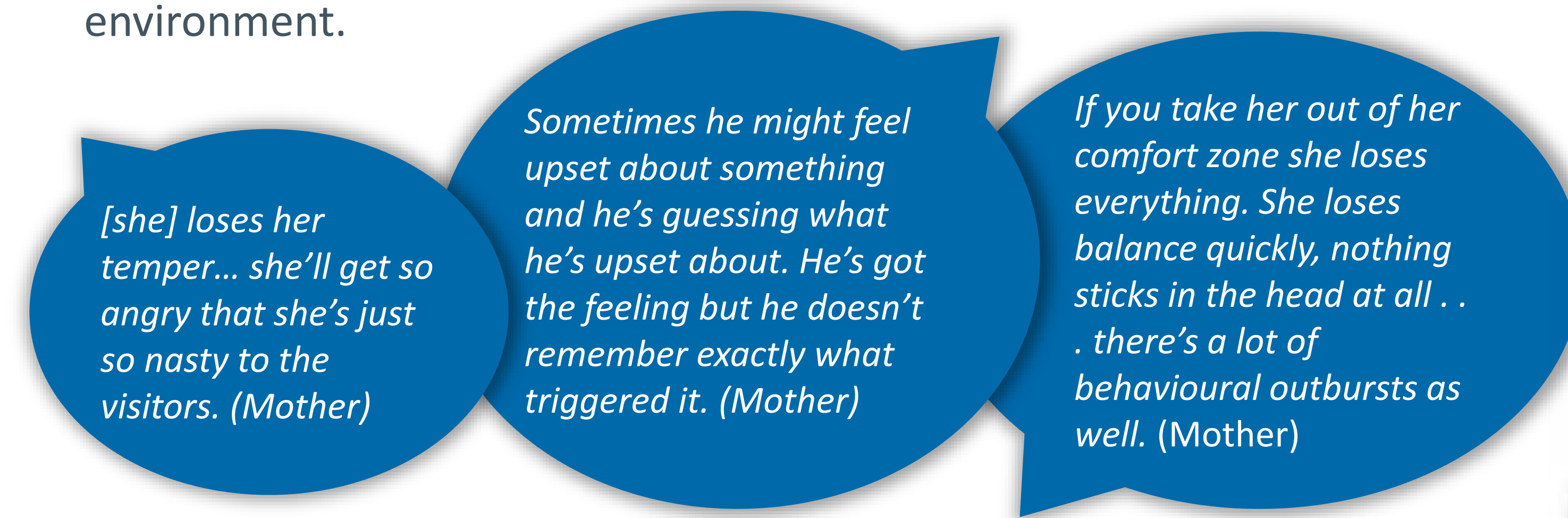

2) What is the impact of challenging behaviours on participation? - Social isolation was seen as one of the most difficult consequences of challenging behavior after TBI. Families reported that their relative with TBI had lost most or all of their old friends and had difficulty forming new friendships due to their behaviours.

- Behaviours such as poor initiation made it difficult for their relative with TB to complete activities of daily living in the home, while their attempts to support or encourage their relative with TBI to complete ADLS could trigger aggression.

- Community activities, whether it be going to the local shops or participating in study, work, or leisure activities, were limited by challenging behaviours.

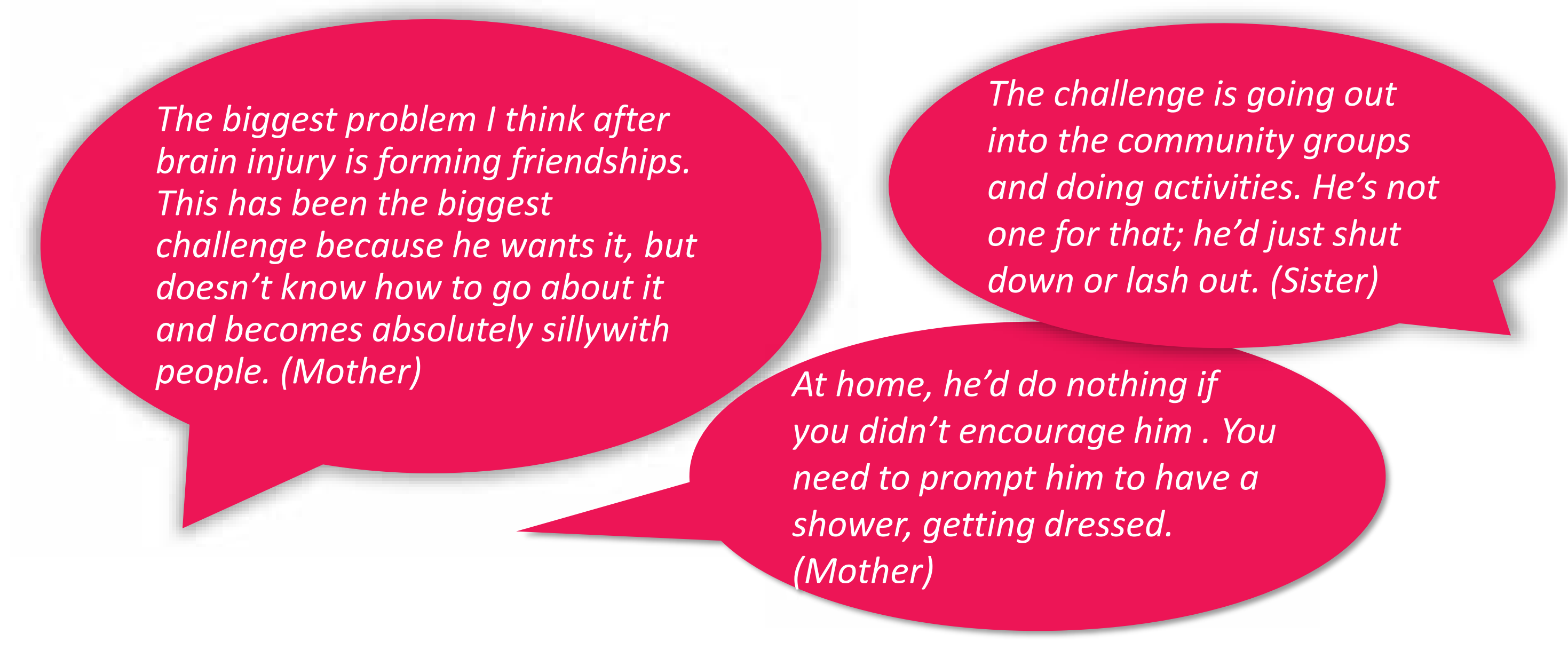

3) How do families support and cope with challenging behaviours?

- Providing support to a relative with challenging behaviours is draining. Family members described issues such as grief (for their family member with TBI but also the broader impact on the family), mental health issues such as anxiety and depression, and in some cases a sense of hopelessnes

Families described a range of ways in which they support their relative with TBI to manage behaviours including: 1) modifying the environment; 2) modifying the demands on the person with $\mathrm{TBl} ; 3$ ) Modifying the way they or other family members interact with the person with TBl; and 4) Giving the person with TBI control over their lives.

- Strategies that helped family members cope in their caring role included: 1) greater acceptance and understanding of the behaviours after TBI; 2 remembering the positives of their situation; and support from 3) socia networks and 4) professionals.

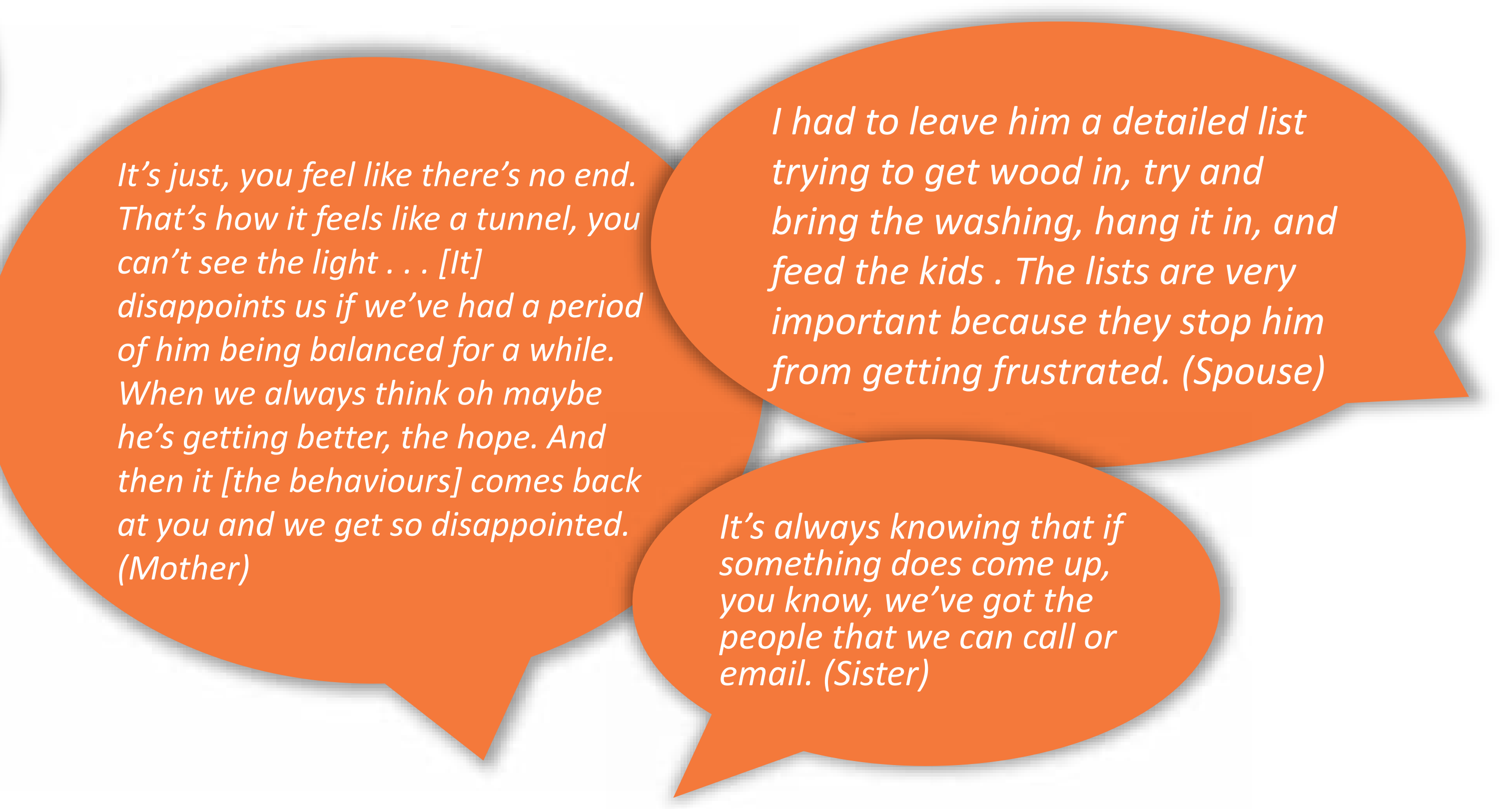

Conclusions

Family conceptualisation of challenging behaviours after TBI may be broader than that of professionals and include associated cognitive problems.

Families consider social isolation as a primary impact of challenging behaviours

Family relatives often have a detailed understanding of challengin behaviours including ways to support the person with TBI

Supporting a relative with challenging behaviours can be draining and so a range of supports for family is important. 